\title{
A meta-analysis and systematic review evaluating the use of erythropoietin in total hip and knee arthroplasty
}

This article was published in the following Dove Press journal: Therapeutics and Clinical Risk Management

Yi Li*

Pengbin Yin*

Houchen Lv

Yutong Meng

Licheng Zhang

Peifu Tang

Department of Orthopedics, General Hospital of Chinese PLA, Beijing,

People's Republic of China

*These authors contributed equally to this work
Correspondence: Licheng Zhang;

Peifu Tang

Department of Orthopedics, General Hospital of Chinese PLA, No. 28 Fuxing Road, Haidian District, Beijing 100853, People's Republic of China

Tel +86 I06693 8I0I; +86 I0 682I 2342

Email pftang301@163.com; zhanglcheng218@I26.com
Purpose: The debate is still ongoing on the effectiveness and safety of erythropoietin (EPO) treatment in orthopedic surgeries. Specifically, previous studies have not compared the dynamic change of hemoglobin $(\mathrm{Hb})$ levels between different transfusion methods. Besides, complications or side effects of this alternative have not been quantitatively analyzed. We conducted a meta-analysis and systemic review to evaluate the efficacy of EPO on $\mathrm{Hb}$ levels observed during the whole perioperative period as well as the volume of allogeneic blood transfusion (ABT), the risk of venous thromboembolism, and application frequency of ABT in hip and knee surgery. Materials and methods: PubMed, Embase, Web of Science, and the Cochrane library were systematically searched from inception to November 2017. The data from randomized controlled trials were extracted and the risk of bias assessed using Cochrane's Collaboration's tool.

Results: Twenty-five randomized controlled trials involving 4,159 patients were included in this meta-analysis. EPO could reduce exposure to allogeneic blood transfused (odds ratio $[\mathrm{OR}]=0.42, P=0.001)$ and reduce the average volume of allogeneic blood transfused $(\mathrm{OR}=-0.28$, $P=0.002)$. When EPO and preoperative autologous blood donation (PABD) were compared, the use of EPO was associated with lower exposure to ABT (OR $=0.48, P=0.03$ ), but no significant decrease in the average volume of allogeneic blood transfused $(\mathrm{OR}=-0.23, P=0.32)$. The use of EPO was associated with a higher level of $\mathrm{Hb}$ with or without use of PABD at all the 4 time points (preoperation, 24-48 hours postoperation, 3-5 days postoperation, discharge of last observation) $(P<0.0001)$, which means EPO could increase the level of Hb significantly during the perioperative period. The results also indicated EPO does not increase the risk of a venous thromboembolism event.

Conclusion: Preoperative administration of EPO was shown to generally increase Hb levels during the whole perioperative period; however, the extent of the positive effects varies with time points. Additionally, EPO minimizes the need for transfusion significantly in patients undergoing hip or knee surgery without increasing the chance of developing thrombotic complications. Therefore, EPO offers an alternative blood management strategy in total hip arthroplasty and total knee arthroplasty.

Keywords: erythropoietin, allogeneic transfusion, hemoglobin, autologous blood donation, total hip arthroplasty, total knee arthroplasty

\section{Introduction}

Nearly every orthopedic surgery is associated with perioperative blood loss, thus necessitating a potential blood transfusion. Given the risk of transfusion reactions and infection transmission, alternatives have been searched to replace or reduce allogeneic red blood cell (RBC) transfusion. Preoperative autologous blood donation (PABD) is one of the alternatives which has been used in different types of surgeries such as total hip 
arthroplasty (THA) and total knee arthroplasty (TKA) to prevent allogeneic transfusion. ${ }^{1}$ However, the application of this procedure is very limited due to the contraindications, which makes it greatly dependent on the health status of the patient. Treatment with erythropoietin (EPO) that is used to increase the level of hemoglobin $(\mathrm{Hb})$ for many patients with internal diseases has been investigated by numerous clinical trials to assess if it could reduce perioperative autologous blood transfusion. However, debate still exists on the effectiveness and safety of this treatment in orthopedic surgeries.

Prior meta-analyses including randomized controlled trials (RCTs) ${ }^{2-4}$ have shown that, in general, application of EPO is beneficial to postoperative allogeneic transfusion reduction as compared to the control group (those treated with placebo). However, 2 main questions that could determine the value of EPO in actual clinical usage still remain unresolved. First, due to limited number of RCTs included by prior meta-analyses, the safety of EPO has not been evaluated comprehensively, which means complications, especially venous thromboembolism (VTE), of this alternative have not been quantitatively analyzed. Second, when determining the efficacy of EPO in reducing the allogeneic transfusion, the main index of evaluation is the requirement for allogeneic blood transfusion (ABT). Few meta-analyses pay close attention to the $\mathrm{Hb}$ levels during the perioperative period, which could directly reflect the severity of anemia. To resolve these questions, we designed this new systematic review and meta-analysis. For the first question, this research included 10 studies; these studies provided more precise data about the occurrence of thromboembolism events in their populations, which made it possible for quantitative analysis. As for the second question, our hypothesis is that monitoring the postoperative $\mathrm{Hb}$ fluctuation, along with the various time intervals, would help to evaluate different transfusion methods precisely. Thus, in addition to pool-estimating the transfusion exposure rate, our meta-analysis placed a focus on whether EPO has effects on $\mathrm{Hb}$ levels at 4 specific time points: preop, 24-48 hours postop, 3-5 days postop, and discharge (last observation). Since the decision on whether a patient requires an $\mathrm{ABT}$ or not significantly relies on the time point during $\mathrm{Hb}$ level is measured, a meta-analysis on effects of EPO on $\mathrm{Hb}$ fluctuation at these specific time points may better define its efficacy in patients.

\section{Materials and methods}

\section{Study selection}

For this systematic review and meta-analysis, PubMed, Embase, Web of Science, and the Cochrane library were systematically searched from inception to November 2017. The following terms were used for searching: erythropoietin, EPO, epoetin alfa, epoetin beta, recombinant human erythropoietin, rHuEPO, THA, total hip replacement, orthopedic surgeries. Two reviewers independently performed the screening of titles, abstract, and full-text articles. Consensus in the selection process was reached through discussion. If consensus was not reached, a third reviewer was consulted.

\section{Inclusion and exclusion criteria}

This systematic review included all adult patients (age $>18$ ) who were eligible for inclusion from studies that reported results of RCTs comparing the effects of EPO in the subjects and controls in an adult population undergoing orthopedic surgeries. The primary outcome was the number of patients exposed to allogeneic transfusion. The secondary outcome included the mean number of allogeneic RBC units transfused and the level of $\mathrm{Hb}$ during the preoperative period. Administration of EPO should start prior to surgery.

Studies that met any one of the following criteria were excluded: 1) duplicates; 2) nonrandomized clinical trials; 3) trials that included surgeries other than orthopedic surgeries.

\section{Quality assessment}

All the included trials were evaluated using Cochrane Collaboration's tool for assessing risk of bias in randomized trials and the Grading of Recommendations Assessment, Development and Evaluation Working Group grading scheme. The risk of bias of the RCTs is demonstrated in Figure 1.

\section{Data extraction}

For each selected trial, the reviewers independently extracted study characteristics, and primary and secondary outcomes. Data shown only in figures or tables were extracted to numerical values, when possible; the data were extracted twice by 2 independent reviewers. After collection, data were checked, once again, and discrepancies were resolved.

\section{Statistical analysis}

Data were analyzed using Review Manager software (RevMan, version 5.3; The Cochrane Collaboration, London, UK). Mean \pm standard deviation was used to calculate the weighted mean difference and 95\% confidence interval.

\section{Results Characteristics of included studies}

The initial literature search identified 180 articles. After removing duplicates, 119 articles were screened based on 


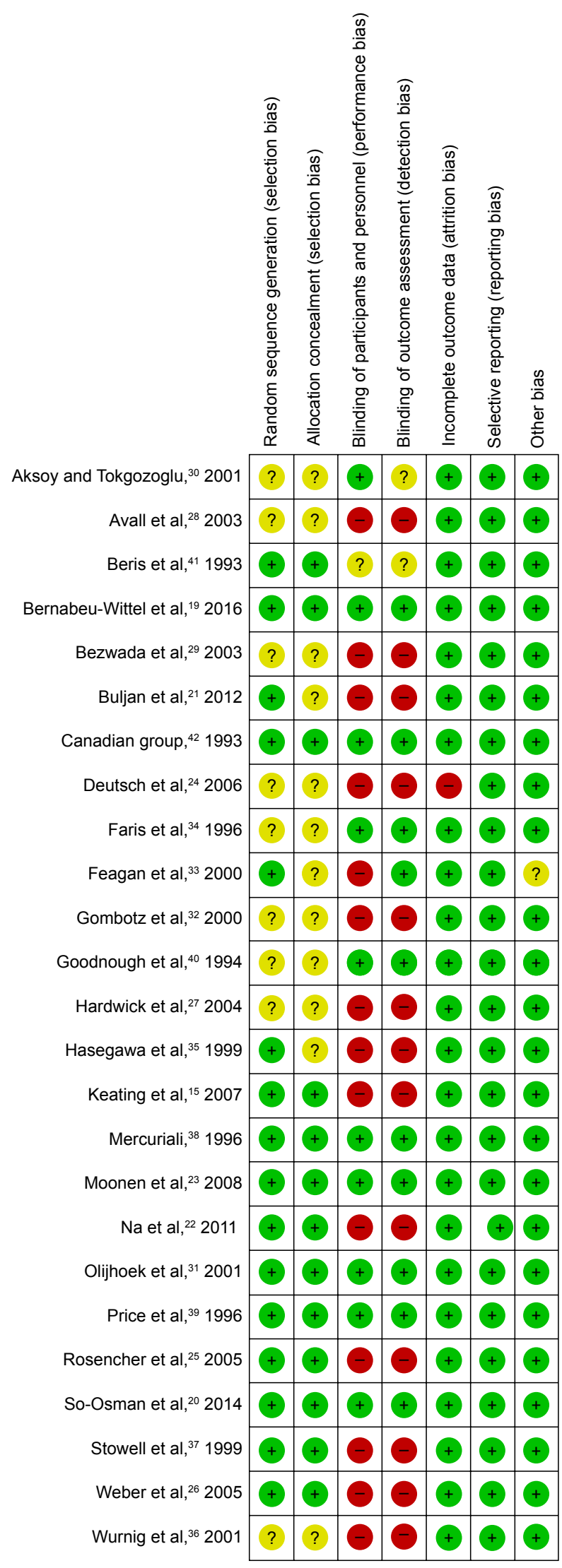

Figure I Risk of bias summary.

Notes: QUADAS-2 was used to analysis the quality of including trials. The green circles mean "Yes." Yellow circles mean "Unsure" and red circles mean "No."

Abbreviation: QUADAS-2, Quality Assessment of Diagnostic Accuracy Studies-2. titles and abstracts, and 71 articles were excluded which were unrelated articles, research about other diseases, or noncontrolled studies. After full-text screening, 23 articles were excluded, 4 of which were from the same data samples, 2 of which were not RCTs and 18 articles did not report desired outcomes (Figure 2). Altogether, 25 RCTs involving 4,159 patients were included in this systematic review and meta-analysis (Table 1). Among these RCTs, 8 trials compared patients that received EPO with those who did not. Eight trials compared patients that received EPO with those who received PABD. Eleven RCTs compared the effect of EPO plus PABD with PABD alone. This meta-analysis divided the trial participants into 3 subgroups: EPO alone versus no EPO, EPO versus PABD, and EPO plus PABD versus PABD alone. QUADAS (Quality Assessment of Diagnostic Accuracy Studies)-2 showed the quality of the including trials. Random sequence generation results showed that 16 studies described a random component in the sequence generation process and 8 studies did not show their sequence generation process. Thirteen studies described methods to conceal allocation, while 11 studies did not describe this clearly. The results showed 14 trials did not blind participants and personnel. Thirteen trials did not blind outcome assessment. Nine trials did not describe the random methods clearly. One research provided incomplete outcome data. All the studies shown, the study protocol, and all of the study's prespecified (primary and secondary) outcomes that are of interest in the review, have been reported in the prespecified way. In conclusion, QUADAS-2 reveals low risk of bias in the included studies (Figure 1).

\section{Requirements for $\mathrm{ABT}$}

Comparing EPO group with the control group, the use of EPO resulted in a lower proportion of patients who needed ABT (odds ratio $[\mathrm{OR}]=0.41, P<0.0001$ ) and lower volumes of allogeneic blood transfused (OR $=-0.45, P=0.0002)$ (Figures 3 and 4). When comparing EPO and PABD, the use of EPO was associated with lower exposure to ABT ( $\mathrm{OR}=0.48, P=0.03$ ), but no significant decrease in the average volume of allogeneic blood transfused (OR = $-0.23, P=0.32$ ). In the subgroup of EPO plus PABD versus PABD, combined application of EPO and PABD leads to a lower proportion of patients who needed $\mathrm{ABT}(\mathrm{OR}=0.42$, $P=0.0001$ ), while injection of EPO caused no significant difference in the average volume of allogeneic blood transfused $(\mathrm{OR}=-0.13, P=0.09)$. After taking all studies into consideration, EPO could reduce the exposure to allogeneic blood transfused $(\mathrm{OR}=0.42, P=0.001)$. Meanwhile, it could also 


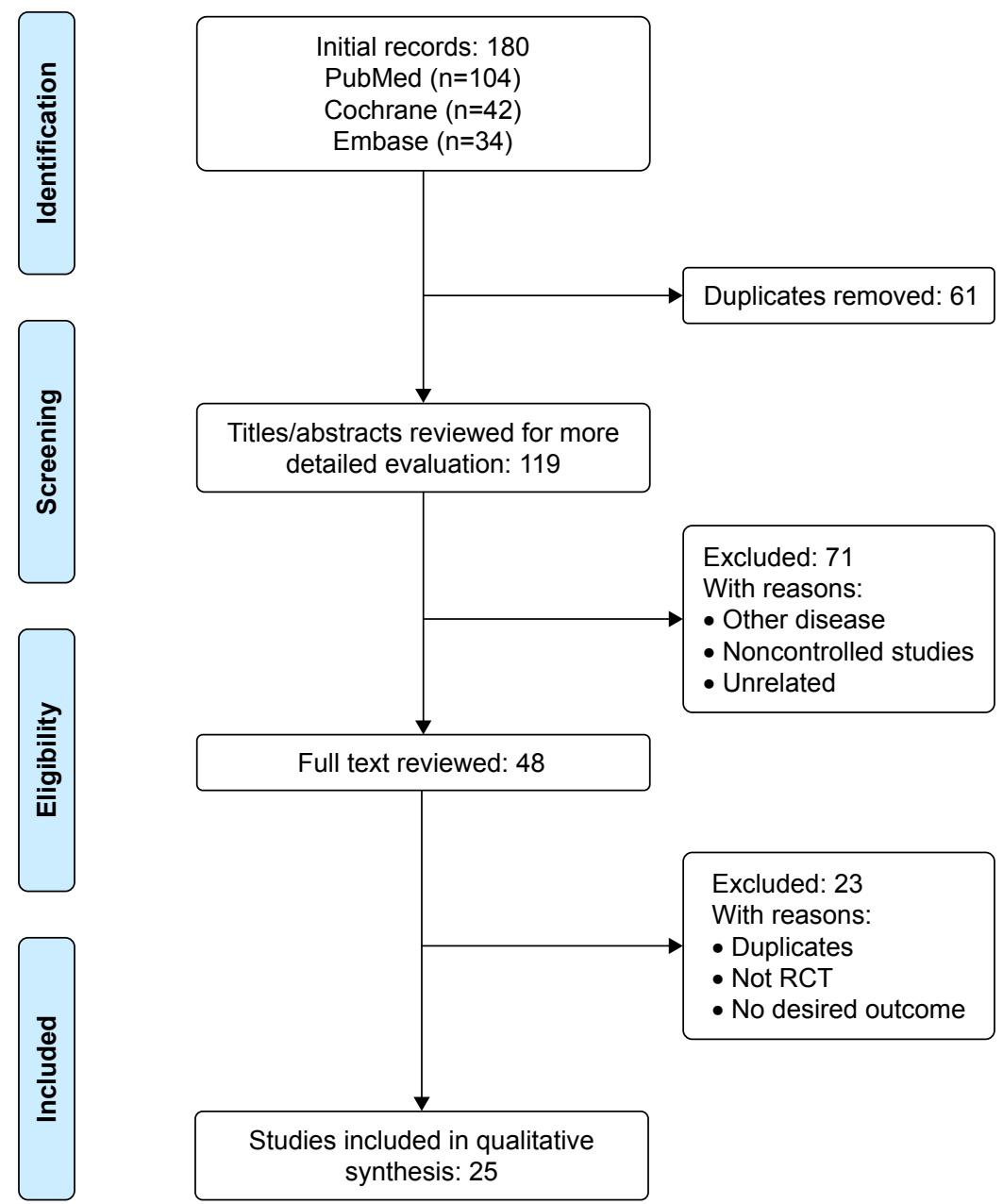

Figure 2 Study flow diagram.

Abbreviation: RCT, randomized controlled trial.

reduce the average volume of allogeneic blood transfused $(\mathrm{OR}=-0.28, P=0.002)$.

\section{$\mathrm{Hb}$ at different time points}

Fifteen trials gave accurate information about the Hb levels during the perioperative period. We extracted and compared the level of $\mathrm{Hb}$ at different times in different subgroups in order to investigate whether the use of EPO could make a dynamic change. We summarized and observed the level of $\mathrm{Hb}$ at 4-time points: preop, $24-48$ hours; postop, 3-5 days postop; and discharge (last observation). No matter what time we tested the value of $\mathrm{Hb}$, using EPO contributed to a higher level of $\mathrm{Hb}$ with or without use of $\mathrm{PABD}(P<0.0001)$, which means EPO could increase the level of $\mathrm{Hb}$ significantly during the perioperative period. All 4 comparisons showed high heterogeneity $\left(I^{2}>50\right)$, which could not be reduced by the preplanned sensitivity analysis to exclude low-quality trials (Figures 5-8).

\section{THA or TKA requirement for allogeneic blood}

To further explore the different effects of EPO in various surgery types, we analyzed the number of patients who had ABTs in the TKA and THA groups. The results showed that there were no significant differences between EPO and control group in TKA $(P=0.07)$ but EPO could reduce the exposure of ABT in THA $(P=0.007)$. These results indicate that more RCTs are needed to figure out whether EPO would have different effects in various surgery types (Figures 9 and 10).

\section{The risk of VTE}

The occurrence of thromboembolism events has only been precisely recorded in 10 trials regarding the use of VTE prophylaxis. Although there were no enough data in the subgroup to compare the risk of VTE in EPO group and PABD group, we put all the studies together and found no 
Table I Characteristics of included studies

\begin{tabular}{|c|c|c|c|c|c|c|}
\hline \multirow[t]{2}{*}{ Study } & \multirow{2}{*}{$\begin{array}{l}\text { Number } \\
\text { of patients }\end{array}$} & \multicolumn{2}{|l|}{ Intervention } & \multirow[t]{2}{*}{ Transfusion criteria } & \multirow{2}{*}{$\begin{array}{l}\text { Transfusion } \\
\text { time }\end{array}$} & \multirow{2}{*}{$\begin{array}{l}\text { Hospital } \\
\text { days }\end{array}$} \\
\hline & & Experimental & Control & & & \\
\hline $\begin{array}{l}\text { Bernabeu- } \\
\text { Wittel et al, }{ }^{19} \\
2016\end{array}$ & 200 & $\begin{array}{l}\text { EPO group: SC single dose of } \\
40,000 \text { IU of EPO }\end{array}$ & $\begin{array}{l}\text { Control: subcutaneous } \\
\text { single-dose placebo } \\
\text { (saline) }\end{array}$ & $\begin{array}{l}\mathrm{Hb}<70 \mathrm{~g} / \mathrm{L} \text {, severe } \\
\text { symptoms plus } \mathrm{Hb} \text { level } \\
\text { between } 7 \mathrm{I} \text { and } 89 \mathrm{~g} / \mathrm{L}\end{array}$ & N/A & 8 days \\
\hline $\begin{array}{l}\text { So-Osman } \\
\text { et } \mathrm{al}^{20}{ }^{20} 2014\end{array}$ & 683 & $\begin{array}{l}\text { EPO group: EPO } 40,000 \text { IU } \\
\text { SC weekly for } 3 \text { weeks before } \\
\text { operation EPO + PABD group: } \\
\text { EPO } 40,000 \text { IU SC weekly for } \\
3 \text { weeks before operation and } \\
\text { autologous blood reinfusion }\end{array}$ & $\begin{array}{l}\text { Control group: } \\
\text { no treatment } \\
\text { PABD group: autologous } \\
\text { blood } \\
\text { Reinfusion }\end{array}$ & N/A & N/A & 8.8 days \\
\hline $\begin{array}{l}\text { Buljan et al, }{ }^{21} \\
2012\end{array}$ & 93 & $\begin{array}{l}\text { EPO group: rHuEPO I5,000 IU } \\
\text { or } 30,000 \text { IU IV twice weekly } \\
\text { for } 3 \text { weeks PABD: I } 2 \% \text { of total } \\
\text { blood volume donated on the } \\
\text { I0th and } 3 \text { rd preoperative days }\end{array}$ & $\begin{array}{l}\text { PABD: } 12 \% \text { of total } \\
\text { blood volume donated } \\
\text { on the } 10 \text { th and } 3 r d \\
\text { preoperative days }\end{array}$ & $\begin{array}{l}\mathrm{Hb} 80 \mathrm{~g} / \mathrm{L} \text { and/or clinical } \\
\text { symptoms of anemia }\end{array}$ & N/A & N/A \\
\hline $\begin{array}{l}\mathrm{Na} \text { et al, } \\
2011\end{array}$ & 108 & $\begin{array}{l}\text { EPO group: rhuEPO- } \beta: 3,000 \text { IU } \\
\text { SC } 3 \text { times }\end{array}$ & $\begin{array}{l}\text { Control group: } \\
\text { no treatment }\end{array}$ & $\mathrm{Hb}<70 \mathrm{~g} / \mathrm{L}$ & N/A & N/A \\
\hline $\begin{array}{l}\text { Moonen } \\
\text { et al, }{ }^{23} 2008\end{array}$ & 100 & $\begin{array}{l}\text { EPO group: EPO- } \alpha \text { : } 40,000 \text { IU } \\
\text { weekly for } 4 \text { weeks }\end{array}$ & $\mathrm{PABD}$ & $\mathrm{N} / \mathrm{A}$ & N/A & N/A \\
\hline $\begin{array}{l}\text { Keating } \\
\text { et al, }{ }^{15} 2007\end{array}$ & 251 & $\begin{array}{l}\text { EPO group: } 600 \mathrm{IU} / \mathrm{kg} \text { weekly } \\
\text { for } 3 \text { weeks and with } 24 \mathrm{~h} \\
\text { postoperatively }\end{array}$ & $\begin{array}{l}\text { PABD: I U before TKA, } \\
2 \cup \text { before THA }\end{array}$ & $\mathrm{Hb}<80 \mathrm{~g} / \mathrm{L}$ & $\mathrm{N} / \mathrm{A}$ & N/A \\
\hline $\begin{array}{l}\text { Deutsch } \\
\text { et al, },^{24} 2006\end{array}$ & 50 & $\begin{array}{l}\text { EPO group: } 40,000 \text { IU SC } \\
\text { I4 days and } 7 \text { days before } \\
\text { operation }\end{array}$ & $\begin{array}{l}\text { PABD group: } 2 \mathrm{U} \text { if } \mathrm{Hb} \\
\text { between } 110 \text { and } 130 \mathrm{~g} / \mathrm{L}\end{array}$ & $\mathrm{Hct}<25 \%$ & N/A & N/A \\
\hline $\begin{array}{l}\text { Rosencher } \\
\text { et al, }{ }^{25} 2005\end{array}$ & 86 & $\begin{array}{l}\text { EPO group: } 40,000 \text { IU SC per } \\
\text { week beginning } 3 \text { weeks before } \\
\text { operation }\end{array}$ & $\begin{array}{l}\text { PABD: once a week } \\
\text { starting } 3 \text { weeks before } \\
\text { surgery, as long as } \\
\mathrm{Hct}>3 \%\end{array}$ & $\begin{array}{l}\text { Hct between } 21 \% \text { and } \\
30 \%\end{array}$ & $\mathrm{~N} / \mathrm{A}$ & N/A \\
\hline $\begin{array}{l}\text { Weber } \\
\text { et al, }{ }^{26} 2005\end{array}$ & 695 & $\begin{array}{l}\text { EPO alfa } 40,000 \text { IU } \\
\text { subcutaneously once weekly for } \\
3 \text { weeks before surgery and on } \\
\text { the day of surgery }\end{array}$ & Placebo & $\mathrm{Hb}<80 \mathrm{~g} / \mathrm{L}$ & N/A & 10.8 days \\
\hline $\begin{array}{l}\text { Hardwick } \\
\text { et al, }{ }^{27} 2004\end{array}$ & 40 & $\begin{array}{l}\text { Epoetin alfa: } 40,000 \text { IU weekly } \\
\text { for } 2 \text { weeks }\end{array}$ & $\begin{array}{l}\text { PABD: I or } 2 \mathrm{U} \text { before } \\
\text { operation }\end{array}$ & N/A & $\mathrm{N} / \mathrm{A}$ & 3.1 days \\
\hline $\begin{array}{l}\text { Avall et al, }{ }^{28} \\
2003\end{array}$ & 23 & $\begin{array}{l}\text { EPO: I } 0,000 \text { IU SC for } 5 \text { times } \\
\text { PABD: I U before } 3,2 \text {, and } \\
\text { I week before surgery }\end{array}$ & $\begin{array}{l}\text { PABD: I U before } 3 \text {, } \\
2 \text { and I week before } \\
\text { surgery }\end{array}$ & $\begin{array}{l}\mathrm{Hb}<85 \mathrm{~g} / \mathrm{L} \text { or when } \\
\text { in danger of inadequate } \\
\text { oxygenation }\end{array}$ & $\mathrm{N} / \mathrm{A}$ & N/A \\
\hline $\begin{array}{l}\text { Bezwada } \\
\text { et al, }{ }^{29} 2003\end{array}$ & 93 & $\begin{array}{l}\text { Epoetin alfa: } 40,000 \text { IU or } \\
20,000 \text { IU SC per week } \\
\text { beginning from } 4 \text { weeks before } \\
\text { operation }\end{array}$ & $\begin{array}{l}\text { PABD: I } \cup \text { for unilateral } \\
\text { arthroplasty and } 2 \cup \text { for } \\
\text { bilateral arthroplasty }\end{array}$ & $\begin{array}{l}\mathrm{Hb}<80 \mathrm{~g} / \mathrm{L} \text { and/or } \\
\text { persistent or } \\
\text { hemodynamically unstable }\end{array}$ & N/A & N/A \\
\hline $\begin{array}{l}\text { Aksoy and } \\
\text { Tokgozoglu, }^{30}\end{array}$ & 40 & $\begin{array}{l}\text { rHuEPO: } 300 \mathrm{lU} / \mathrm{kg} \text { twice a } \\
\text { week for } 2 \text { weeks, then once }\end{array}$ & PABD & $\begin{array}{l}\mathrm{Hb}<80 \mathrm{~g} / \mathrm{L} \text { or } \\
\text { hemodynamically unstable }\end{array}$ & N/A & N/A \\
\hline 2001 & & $\begin{array}{l}3 \text { days before operation PABD: } \\
\text { I unit at } 4 \text { days interval until } \\
\mathrm{Hb}<100 \mathrm{~g} / \mathrm{L}\end{array}$ & & & & \\
\hline $\begin{array}{l}\text { Olijhoek } \\
\text { et al, },^{31} 200 \text { I }\end{array}$ & 110 & $\begin{array}{l}\text { Epoetin alfa: } 600 \mathrm{IU} / \mathrm{kg} \text { weekly } \\
\text { for } 3 \text { weeks }\end{array}$ & Placebo & $\mathrm{N} / \mathrm{A}$ & N/A & N/A \\
\hline $\begin{array}{l}\text { Gombotz } \\
\text { et al, }{ }^{32} 2000\end{array}$ & 40 & $\begin{array}{l}\text { rHuEPO: } 600 \mathrm{U} / \mathrm{kg} \text { SC on day } \\
14 \text { and, if needed, on day } 7 \\
\text { before surgery }\end{array}$ & $\begin{array}{l}\text { PABD: starting } 4 \text { weeks } \\
\text { before surgery (goal: } 3 \mathrm{U} \\
\text { per donor) }\end{array}$ & $\mathrm{N} / \mathrm{A}$ & $N / A$ & N/A \\
\hline $\begin{array}{l}\text { Feagan } \\
\text { et al, },^{33} 2000\end{array}$ & 160 & $\begin{array}{l}\text { Epoetin alfa: } 40,000 \text { IU or } \\
20,000 \text { IU SC per week } \\
\text { beginning from } 4 \text { weeks before } \\
\text { operation }\end{array}$ & Placebo & $\mathrm{N} / \mathrm{A}$ & $\mathrm{N} / \mathrm{A}$ & N/A \\
\hline
\end{tabular}


Table I (Continued)

\begin{tabular}{|c|c|c|c|c|c|c|}
\hline \multirow[t]{2}{*}{ Study } & \multirow{2}{*}{$\begin{array}{l}\text { Number } \\
\text { of patients }\end{array}$} & \multicolumn{2}{|l|}{ Intervention } & \multirow[t]{2}{*}{ Transfusion criteria } & \multirow{2}{*}{$\begin{array}{l}\text { Transfusion } \\
\text { time }\end{array}$} & \multirow{2}{*}{$\begin{array}{l}\text { Hospital } \\
\text { days }\end{array}$} \\
\hline & & Experimental & Control & & & \\
\hline Faris et al, ${ }^{34}$ & 185 & I. EPO (300 U/kg) daily or & Placebo & $\mathrm{N} / \mathrm{A}$ & $N / A$ & $\mathrm{~N} / \mathrm{A}$ \\
\hline 1996 & & 2. EPO $100 \mathrm{U} / \mathrm{kg}$ daily & & & & \\
\hline $\begin{array}{l}\text { Hasegawa } \\
\text { et al, }{ }^{35} 1999\end{array}$ & 37 & Epoetin beta: $6,000 \mathrm{U}+\mathrm{PABD}$ & $\begin{array}{l}\text { PABD group: autologous } \\
\text { blood reinfusion }\end{array}$ & N/A & N/A & N/A \\
\hline $\begin{array}{l}\text { Wurnig } \\
\text { et al, }{ }^{36} 200 \text { I }\end{array}$ & 110 & Epoetin beta: $250 \mathrm{IU} / \mathrm{kg}$ & Placebo & $\mathrm{Hb}<85 \mathrm{~g} / \mathrm{L}$ & $\mathrm{N} / \mathrm{A}$ & $\mathrm{N} / \mathrm{A}$ \\
\hline $\begin{array}{l}\text { Stowell } \\
\text { et al, }{ }^{37} 1999\end{array}$ & 428 & $\begin{array}{l}\text { Weekly doses of SC EPO alfa } \\
(600 \mathrm{U} / \mathrm{kg}) \text { on preoperative } \\
\text { days: } 2 \mathrm{I}, 14 \text {, and } 7 \text {, and on the } \\
\text { day of surgery }\end{array}$ & PABD & N/A & N/A & $\mathrm{N} / \mathrm{A}$ \\
\hline $\begin{array}{l}\text { Mercuriali, }{ }^{38} \\
1996\end{array}$ & 44 & $\begin{array}{l}\mathrm{EPO} \text { at } 600 \mathrm{U} / \mathrm{kg} ; \text { patients } \\
\text { received } \mathrm{EPO} \text { at } 300 \mathrm{U} / \mathrm{kg}\end{array}$ & Placebo & $\mathrm{Hct}<34 \%$ & N/A & $\mathrm{N} / \mathrm{A}$ \\
\hline $\begin{array}{l}\text { Price et al, }{ }^{39} \\
1996\end{array}$ & 173 & $\mathrm{EPO}$ at $600 \mathrm{U} / \mathrm{kg}$ & PABD & N/A & $\mathrm{N} / \mathrm{A}$ & $\mathrm{N} / \mathrm{A}$ \\
\hline $\begin{array}{l}\text { Goodnough } \\
\text { et al, }{ }^{40} 1994\end{array}$ & 91 & $\begin{array}{l}\text { Patients received EPO at } \\
600 \mathrm{U} / \mathrm{kg} ; \text { patients received } \\
\mathrm{EPO} \text { at } 300 \mathrm{U} / \mathrm{kg}\end{array}$ & Placebo & Hct $<33 \%$ & $\mathrm{~N} / \mathrm{A}$ & N/A \\
\hline $\begin{array}{l}\text { Beris et al, }{ }^{41} \\
1993\end{array}$ & 101 & $\begin{array}{l}\text { EPO I } 50-180 \mathrm{U} / \mathrm{kg}(10,000 \mathrm{U}) \\
\text { SC given } 3 \text { times per week: } \\
4 \text { and } 2 \text { weeks before surgery }\end{array}$ & PABD & N/A & N/A & N/A \\
\hline $\begin{array}{l}\text { Canadian } \\
\text { group, }{ }^{42} 1993\end{array}$ & 218 & $\begin{array}{l}\text { I. I } 4 \text { days of EPO }(300 \mathrm{U} / \mathrm{kg} \text { to } \\
\text { a maximum of } 30,000 \mathrm{U}) \\
\text { or } \\
\text { 2. EPO for the } 9 \text { days after } \\
\text { placebo }\end{array}$ & Placebo & $\mathrm{Hb}<90 \mathrm{~g} / \mathrm{L}$ & N/A & $\mathrm{N} / \mathrm{A}$ \\
\hline Total & 4,159 & & & & & \\
\hline
\end{tabular}

Abbreviations: EPO, erythropoietin; Hb, hemoglobin; Hct, hematocrit; IV, intravenous; N/A, not applicable; PABD, preoperative autologous blood donation; rHuEPO, recombinant human erythropoietin; SC, subcutaneous; THA, total hip arthroplasty; TKA, total knee arthroplasty.

significant differences with or without use of EPO $(P=0.17)$. As a result, we can conclude that EPO does not increase the risk of a VTE event (Figure 11).

\section{Discussion}

EPO is a glycoprotein hormone produced by the kidney which regulates erythropoiesis by stimulating the EPO receptors. Recombinant human EPO has been used successfully for over 20 years to treat anemia in millions of patients. As anemia is a common feature of many diseases, erythropoiesisstimulating agents have been potential therapeutic strategies for anemia management. Subjects with chronic kidney disease (CKD) often develop anemia because of decreased production of EPO resulting in insufficient erythropoiesis. As anemia is a significant, prognostic parameter in the development of CKD, the treatment of anemia in CKD patients by stimulating erythropoiesis with recombinant human EPO or other erythropoiesis-stimulating agents will be of great importance. 5,6 Although EPO has been used to treat renal anemia for nearly 2 decades, debate persists over the optimal target $\mathrm{Hb}$ level. Erythropoiesis-stimulating agent studies have shown that augmentation of $\mathrm{Hb}$ to $10-11 \mathrm{~g} / \mathrm{dL}$ is associated with clinical advantages. However, it has not been proven that intensified $\mathrm{Hb}>11 \mathrm{~g} / \mathrm{dL}$ utilizing exogenic EPO translates into a comprehensive benefit for the patients. ${ }^{5,7} \mathrm{New}$ developments promise interesting therapeutic options not only by stimulating erythropoiesis but also by modulating additional mechanisms. In addition to erythropoiesis, EPO has also been reported to have other effects, such as tissue protection and neuroprotection. EPO has demonstrated neuroprotective effects in spinal cord ischemia. EPO-mediated induction of the CREB pathway and production of neurotrophins is associated with improved neurologic function and increased neuronal viability following spinal cord ischemia/ reperfusion. ${ }^{8-10}$ Besides, preclinical studies indicated that EPO may improve outcome after traumatic brain injuries, including in neuroprotection and anemia treatment. ${ }^{11}$ However, clinical trials do not support this view well. EPO did not reduce the number of patients with severe neurological dysfunction (GOS-E level 1-4) or increase the incidence of deep venous thrombosis of the lower limbs. Furthermore, the effect of EPO on mortality remains uncertain. In sum, 


\begin{tabular}{|c|c|c|c|c|c|c|c|c|c|}
\hline $\begin{array}{l}\text { Study or } \\
\text { subgroup }\end{array}$ & $\begin{array}{l}\text { EPO } \\
\text { events }\end{array}$ & Total & $\begin{array}{l}\text { Control } \\
\text { events }\end{array}$ & Total & $\begin{array}{l}\text { Weight } \\
\text { (\%) }\end{array}$ & $\begin{array}{l}\text { OR } \mathrm{M}-\mathrm{H}, \\
\text { random, } 95 \% \mathrm{Cl}\end{array}$ & $\begin{array}{l}\text { OR } \mathrm{M}-\mathrm{H} \text {, } \\
\text { random, }\end{array}$ & -, $95 \% \mathrm{Cl}$ & \\
\hline EPO vs no EPO & & & & & & & & & \\
\hline Bernabeu-Wittel et al, ${ }^{19} 2016$ & 52 & 100 & 54 & 100 & 5.6 & $0.92(0.53,1.61)$ & & - & \\
\hline Canadian group, ${ }^{42} 1993$ & 34 & 130 & 34 & 78 & 5.4 & $0.46(0.25,0.83)$ & & & \\
\hline Faris et al, ${ }^{34} 1996$ & 25 & 118 & 36 & 67 & 5.1 & $0.23(0.12,0.44)$ & 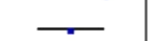 & & \\
\hline Feagan et al, ${ }^{33} 2000$ & 18 & 79 & 35 & 78 & 4.9 & $0.36(0.18,0.72)$ & 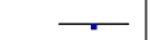 & & \\
\hline Na et al, ${ }^{22} 2011$ & 11 & 54 & 29 & 54 & 4.2 & $0.22(0.09,0.52)$ & $\longrightarrow$ & & \\
\hline So-Osman et al, ${ }^{20} 2014$ & 13 & 125 & 32 & 138 & 4.9 & $0.38(0.19,0.77)$ & 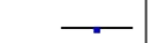 & & \\
\hline Wurnig et al, ${ }^{36} 2001$ & 22 & 59 & 28 & 51 & 4.6 & $0.49(0.23,1.05)$ & & & \\
\hline Subtotal $(95 \% \mathrm{Cl})$ & & 665 & & 566 & 34.8 & $0.41(0.28,0.60)$ & & & \\
\hline $\begin{array}{l}\text { Total events } \\
\text { Heterogeneity: } \tau^{2}=0.15 ; \chi^{2}=13\end{array}$ & $\begin{array}{l}175 \\
57, d f=6\end{array}$ & $D=0.03)$ & $\begin{array}{l}248 \\
l^{2}=56 \%\end{array}$ & & & & & & \\
\hline Test for overall effect: $Z=4.57$ ( & $><0.0000$ & & & & & & & & \\
\hline EPO vs PABD & & & & & & & & & \\
\hline Bezwada et al, ${ }^{29} 2003$ & 22 & 80 & 26 & 80 & 5.0 & $0.79(0.40,1.55)$ & & - & \\
\hline Deutsch et al, ${ }^{24} 2006$ & 7 & 25 & 2 & 25 & 1.8 & $4.47(0.83,24.19)$ & & & \\
\hline Gombotz et al, ${ }^{32} 2000$ & 6 & 20 & 8 & 20 & 2.6 & $0.64(0.17,2.38)$ & & & \\
\hline Hardwick et al, ${ }^{27} 2004$ & 2 & 19 & 3 & 21 & 1.5 & $0.71(0.10,4.76)$ & & & \\
\hline Keating et al, ${ }^{15} 2007$ & 4 & 130 & 17 & 121 & 3.2 & $0.19(0.06,0.60)$ & & & \\
\hline Moonen et al, ${ }^{23} 2008$ & 2 & 50 & 14 & 50 & 2.1 & $0.11(0.02,0.50)$ & & & \\
\hline Rosencher et al, ${ }^{25} 2005$ & 3 & 45 & 5 & 41 & 2.2 & $0.51(0.11,2.30)$ & & & \\
\hline So-Osman et al, ${ }^{20} 2014$ & 13 & 125 & 60 & 206 & 5.1 & $0.28(0.15,0.54)$ & & & \\
\hline Subtotal $(95 \% \mathrm{Cl})$ & & 494 & & 564 & 23.5 & $0.48(0.25,0.91)$ & & & \\
\hline $\begin{array}{l}\text { Total events } \\
\text { Heterogeneity: } \tau^{2}=0.48 ; \gamma^{2}=17\end{array}$ & $\begin{array}{l}59 \\
77, d f=7\end{array}$ & $D=0.01)$ & $\begin{array}{l}135 \\
l^{2}=61 \%\end{array}$ & & & & & & \\
\hline Test for overall effect: $Z=2.23$ ( & $D=0.03)$ & & & & & & & & \\
\hline EPO + PABD vs PABD & & & & & & & & & \\
\hline Aksoy and Tokgozoglu, ${ }^{30} 2001$ & 5 & 20 & 9 & 20 & 2.5 & $0.41(0.11,1.56)$ & & - & \\
\hline Avall et al, ${ }^{28} 2003$ & 7 & 19 & 5 & 19 & 2.4 & $1.63(0.41,6.51)$ & & & \\
\hline Beris et al, ${ }^{41} 1993$ & 3 & 49 & 7 & 52 & 2.4 & $0.42(0.10,1.72)$ & & - & \\
\hline Bezwada et al, ${ }^{29} 2003$ & 9 & 80 & 26 & 80 & 4.3 & $0.26(0.11,0.61)$ & & & \\
\hline Buljan et al, ${ }^{21} 2012$ & 6 & 61 & 11 & 32 & 3.2 & $0.21(0.07,0.63)$ & & & \\
\hline Goodnough et al, ${ }^{40} 1994$ & 6 & 68 & 2 & 23 & 1.8 & $1.02(0.19,5.43)$ & & & \\
\hline Mercuriali, ${ }^{38} 1996$ & 9 & 36 & 4 & 8 & 2.0 & $0.33(0.07,1.61)$ & & - & \\
\hline Price et al, ${ }^{39} 1996$ & 17 & 86 & 27 & 87 & 4.9 & $0.55(0.27,1.10)$ & & & \\
\hline So-Osman et al, ${ }^{20} 2014$ & 41 & 214 & 60 & 206 & 6.1 & $0.58(0.37,0.91)$ & & & \\
\hline Stowell et al, ${ }^{37} 1999$ & 27 & 209 & 42 & 219 & 5.8 & $0.63(0.37,1.06)$ & & & \\
\hline Weber et al, ${ }^{26} 2005$ & 41 & 460 & 87 & 235 & 6.3 & $0.17(0.11,0.25)$ & 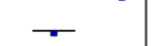 & & \\
\hline Subtotal $(95 \% \mathrm{Cl})$ & & 1,302 & & 981 & 41.7 & $0.42(0.27,0.66)$ & & & \\
\hline Total events & 171 & & 280 & & & & & & \\
\hline Heterogeneity: $\tau^{2}=0.31 ; \chi^{2}=30$ & $38, d f=1 c$ & D-0 C & $77 \cdot 1^{2}=67$ & & & & & & \\
\hline Test for overall effect: $Z=3.84$ & $\mathrm{D}=0.0001)$ & & & & & & & & \\
\hline Total $(95 \% \mathrm{Cl})$ & & 2,461 & & 2,111 & 100 & $0.42(0.33,0.55)$ & & & \\
\hline $\begin{array}{l}\text { Total events } \\
\text { Heterogeneity: } \tau^{2}=0.24 ; \chi^{2}=62\end{array}$ & $\begin{array}{l}405 \\
54, d f=25\end{array}$ & $(P<0.00$ & $\begin{array}{l}663 \\
01) ; I^{2}=60\end{array}$ & & & & & & \\
\hline Test for overall effect: $Z=6.42$ ( & $0<0.0000$ & & & & & 0.01 & 0.1 & 10 & 100 \\
\hline Test for subgroup differences: $\chi$ & $=0.16, d$ & $=2(P=$ & 0) & & & Fav & xperimental) & Favors ( & (control) \\
\hline
\end{tabular}

Figure 3 Patients needing ABT.

Abbreviations: ABT, allogeneic blood transfusion; EPO, erythropoietin; M-H, Mantel-Haenszel; OR, odds ratio; PABD, preoperative autologous blood donation.

more studies are needed to clarify therapeutic mechanism and of EPO.

TKA and THA are associated with considerable blood loss and the subsequent need for transfusions. ${ }^{12}$ Since recipients of allogeneic RBC transfusions remain at risk for febrile, allergic transfusion reactions and potentially high rates of postoperative infection $;{ }^{13}$ clinicians have been seeking a bloodless preoperative therapeutic modality aimed at improving clinical outcomes by reducing these adverse effects. PABD is a way to avoid ABT, but approximately $44 \%$ of the predonated autologous units are discarded ${ }^{14}$ and roughly $14 \%$ of patients who predonate still require ABTs. ${ }^{15}$ Various studies report that this is an effective method to increase preoperative $\mathrm{Hb}$ levels and reduce the frequency of transfusions following 


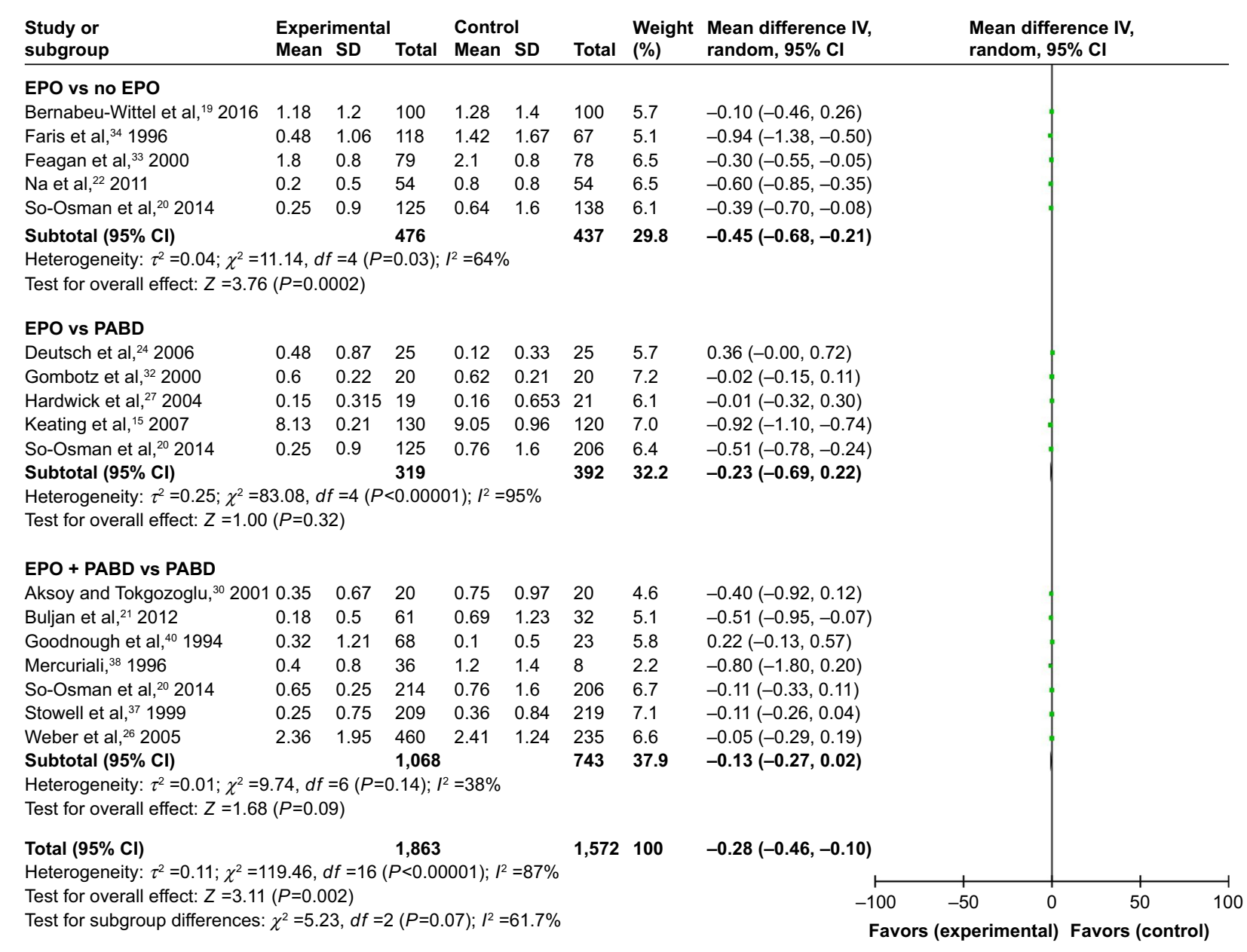

Figure 4 Units of allogeneic blood transfused.

Abbreviations: EPO, erythropoietin; PABD, preoperative autologous blood donation.

TKA. However, evidence for the exclusive use of one of these preoperative blood management strategies individually is controversial. In this study, we analyzed the subgroups to evaluate the effectiveness and safety of EPO including EPO versus no EPO, with or without PABD. The results indicated that the preoperative use of EPO in patients undergoing THA or TKA could significantly reduce the number of patients who were exposed to ABT, with or without PABD. Comparing EPO to PABD, EPO could reduce the number of patients undergoing $\mathrm{ABT}$ more efficiently $(P=0.03)$. The results show significant differences between previous studies. ${ }^{2} \mathrm{We}$ also analyze the units of ABT from different groups. The units of ABT could be reduced by using EPO alone as compared to control group (placebo). However, there was no significant difference between using EPO compared to $\operatorname{PABD}(P=0.32)$. The combined use of EPO and PABD showed no significant difference from using PABD alone $(P=0.09)$. Another important outcome of this meta-analysis was the evaluation of $\mathrm{Hb}$ levels at different time points. We collected data on the $\mathrm{Hb}$ levels before operation, in the early postoperative stage (24-48 hours postoperation), late postoperative stage (3-5 days postoperation), and at discharge/last observation. The analysis showed that EPO could increase $\mathrm{Hb}$ levels at different times among all subgroups with or without PABD. These results indicated that EPO should be recommended during the preoperative period. Preoperative anemia has been associated with increased risk of ABT and postoperative morbidity and mortality. ${ }^{16}$ The higher level of $\mathrm{Hb}$ after surgery may avoid the risk of anemia, shock, myocardial infarctions, and other adverse effect. ${ }^{17}$

This systematic review and meta-analysis collected RCTs published from inception to November 2017 by searching PubMed, Embase, Web of Science, and the Cochrane library. The subjects included in this study were 18-85 year old males and females. The protocols of EPO and PABD are displayed in Table 1. All of our included studies were RCTs. According 


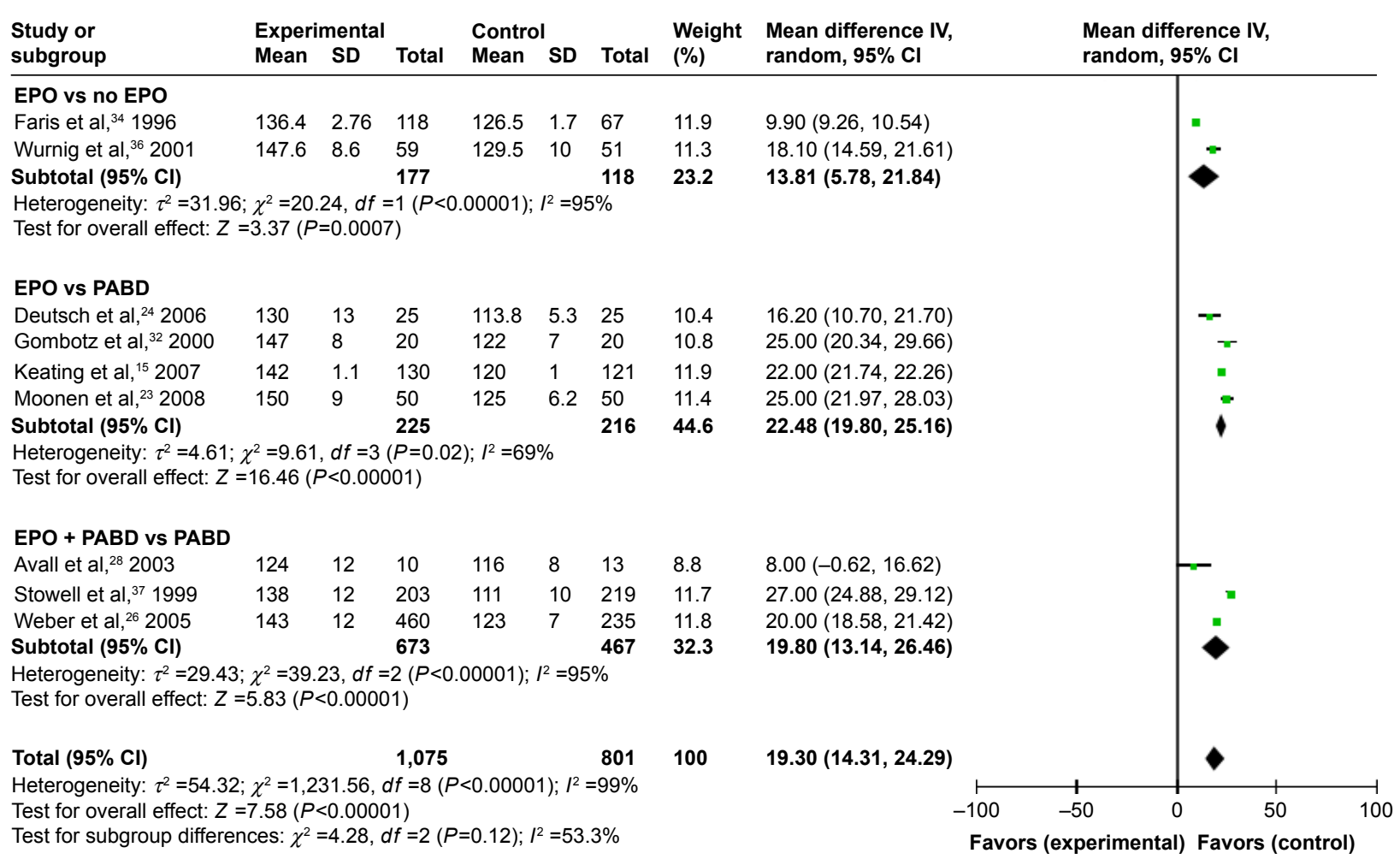

Figure 5 Preoperative $\mathrm{Hb}$.

Abbreviations: EPO, erythropoietin; $\mathrm{Hb}$, hemoglobin; PABD, preoperative autologous blood donation.

to the comparison of the baseline data of the 2 groups, for example, age, gender, etc., the results show comparability between the groups. Thus, we know that at least these factors are not predisposing factors. Whether there are any factors that have not been reported is currently unknown. However, it is certain that EPO has the effect of promoting erythropoiesis and has applications in many diseases. Therefore, the positive impact of EPO in orthopedic surgery patients can be explained by the effect of EPO. In reviewing these clinical studies, we discovered several experimental design deficiencies. Through this article, we hope that future research will focus on and improve the following aspects. First, the health status of the population included should be assessed more precisely. As mentioned above, due to the potential therapeutic effect of EPO on anemia-induced factors such as diabetes, nephropathy, and neurological diseases, patients with these diseases should also be excluded. Second, endogenous EPO may be a very important indicator which may impact the therapeutic effect of exogenous EPO. However, current studies lack data on monitoring of endogenous EPO. In subsequent studies, endogenous EPO values should be included as part of the inclusion criteria to reduce selection bias. Third, there are some risk factors that have been recognized that greatly increase the risk of VTE in patients, such as advanced age, prolonged braking (paralysis), and malignancy. Several studies have confirmed that the increase in age is associated with an increased risk of VTE. Patients over 40 years of age are at significantly increased risk in developing VTE compared with younger patients, and risk approximately double with each subsequent decade. ${ }^{18}$ There are other factors that reduce the incidence of VTE, such as prophylactic anticoagulant therapy. On account of the low rate of VTE that occurred during the perioperative period, we only summarized 8 trials, which explicitly reported that VTE occurred during these studies but that there was no difference between EPO and the control groups $(P=0.20)$. However, the main factor affecting VTE is preventive anticoagulant therapy. As most patients would receive anticoagulant prophylactic treatment during the perioperative period, the incidence of VTE may therefore decline. As the included studies spanned from 1993 to 2016, the use of prophylactic anticoagulants would have also been changed. Therefore, the risk of VTE may also decrease with the improvement of anticoagulant drugs, thus masking the potential induced risk of EPO. Subsequent RCT studies need to provide detailed explanations of anticoagulation methods in order to better analyze the issue of VTE. 


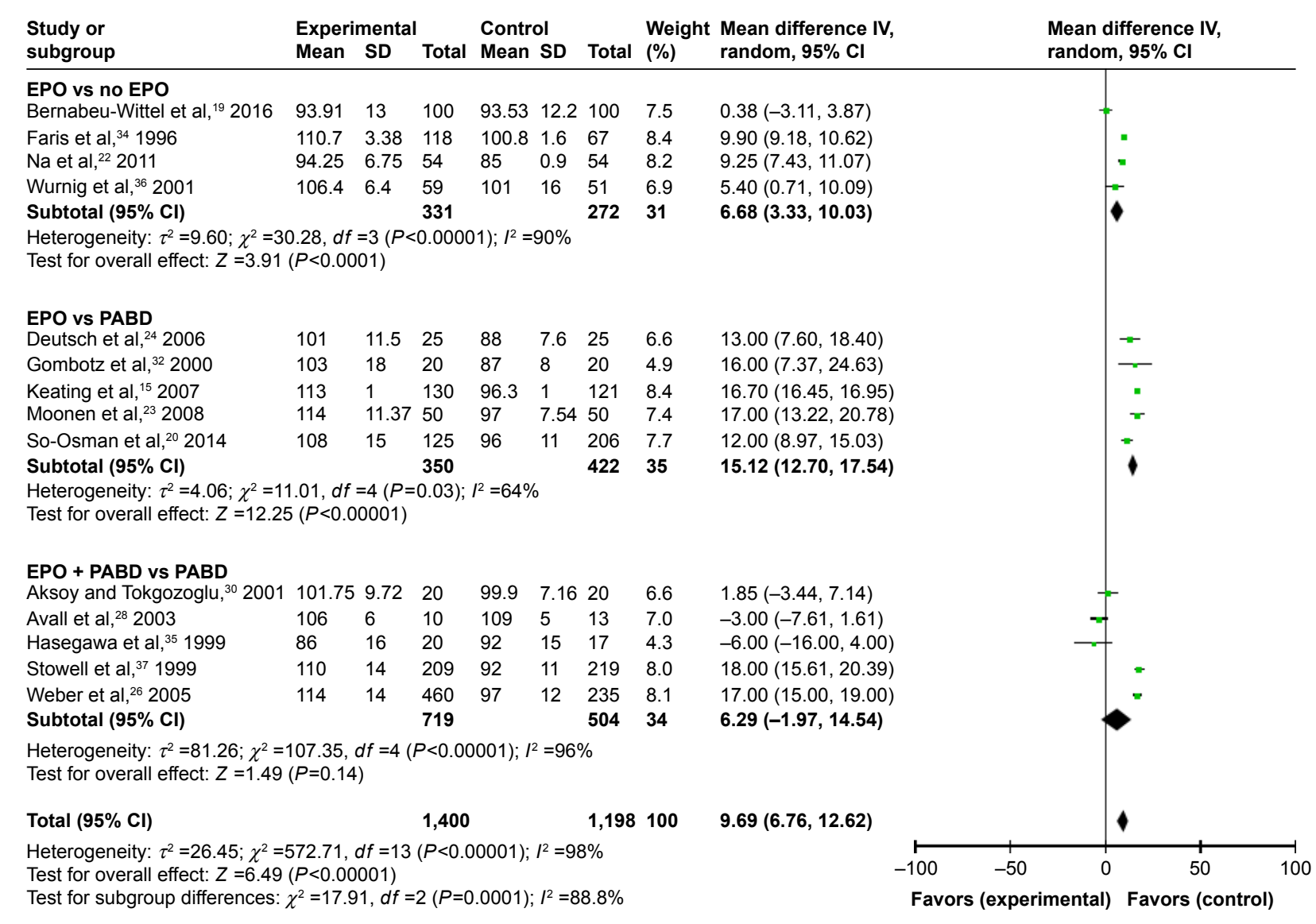

Figure $6 \mathrm{Hb}$ levels 24-48 hours postsurgery.

Abbreviations: EPO, erythropoietin; PABD, preoperative autologous blood donation.

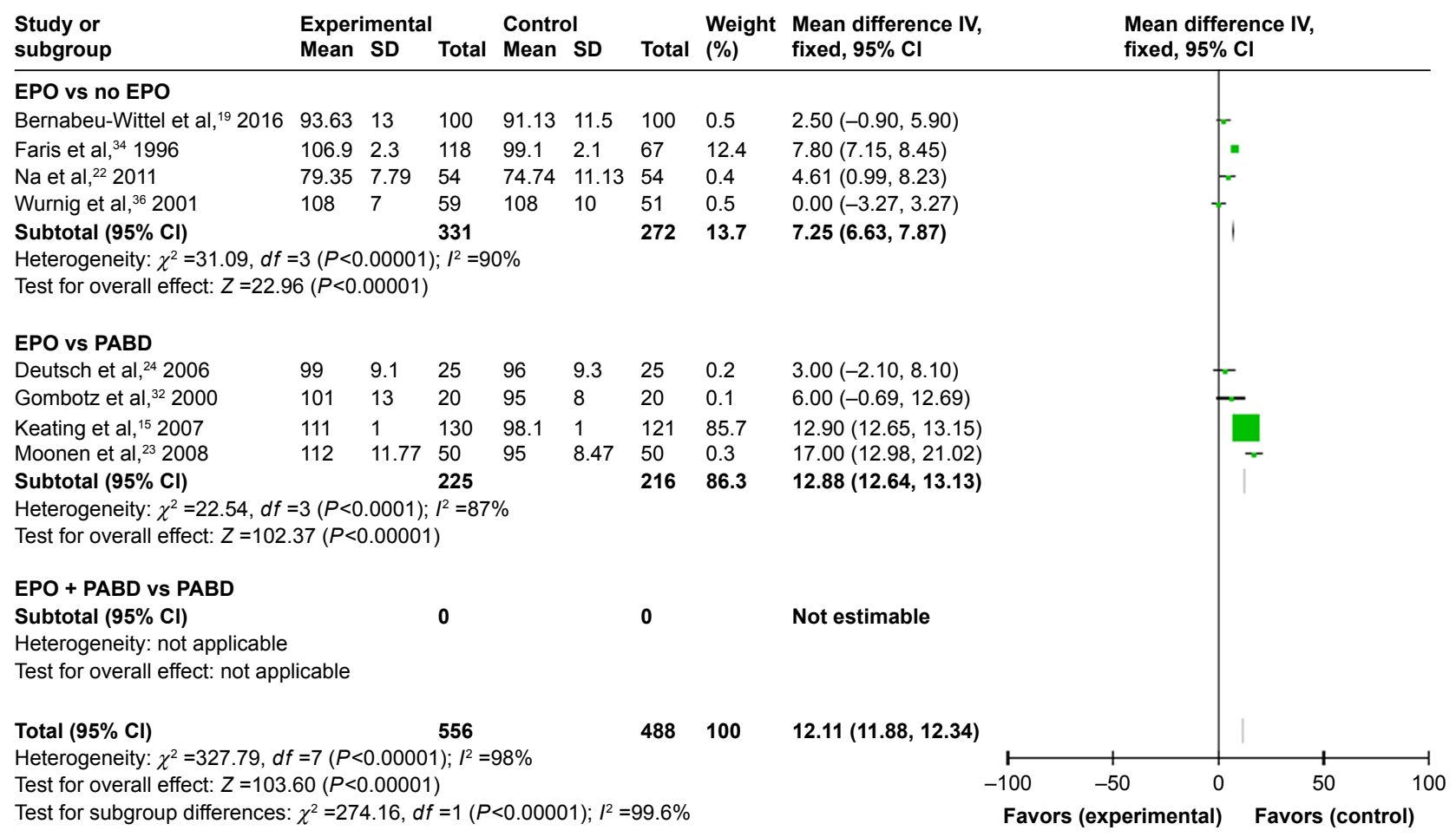

Figure $7 \mathrm{Hb}$ levels $3-5$ days postsurgery.

Abbreviations: EPO, erythropoietin; Hb, hemoglobin; PABD, preoperative autologous blood donation. 


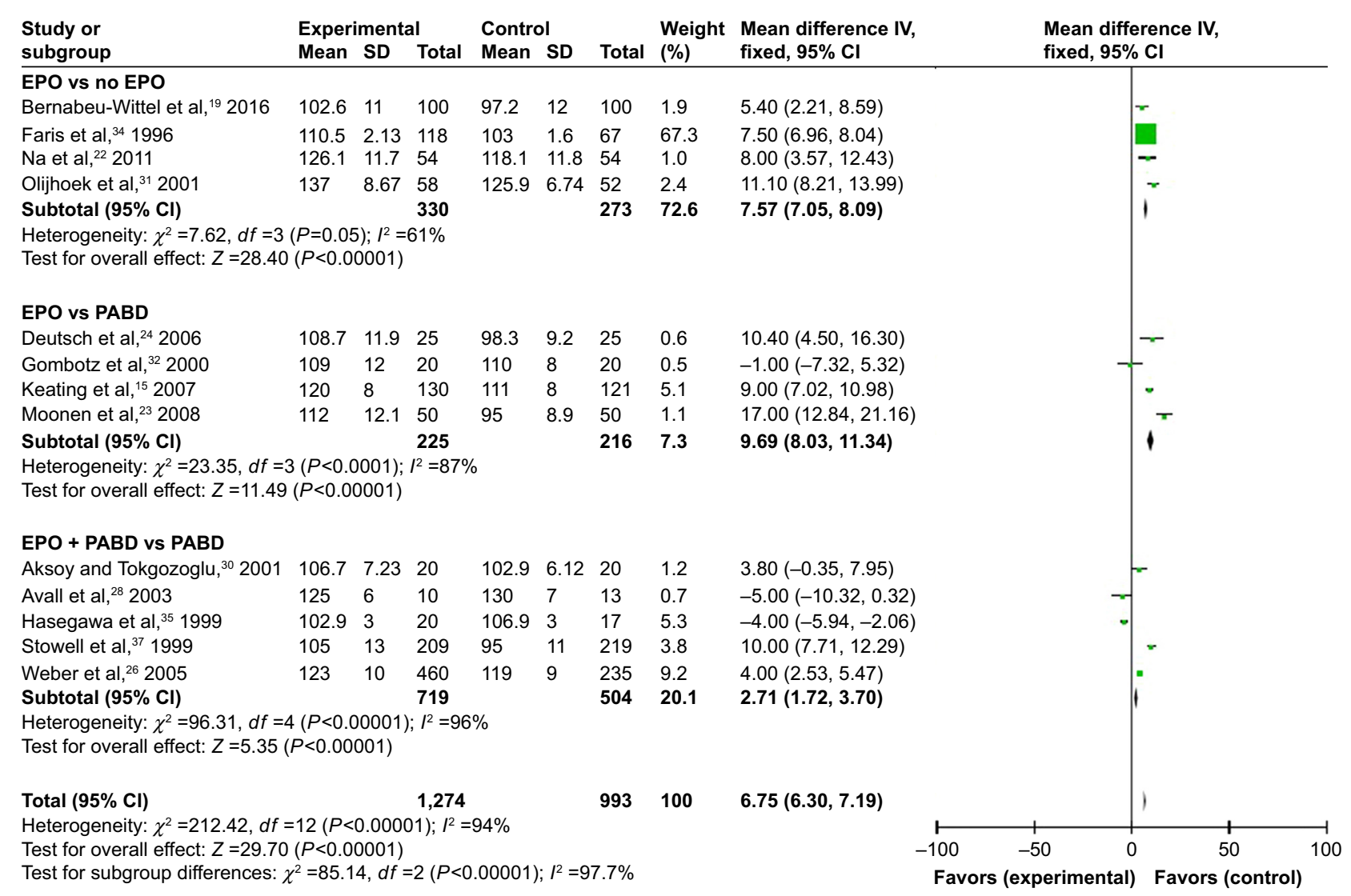

Figure $8 \mathrm{Hb}$ level at discharge/last observation.

Abbreviations: EPO, erythropoietin; Hb, hemoglobin; PABD, preoperative autologous blood donation.

Combining this study with past related research, we propose some suggestions. The administration of EPO would improve patients' symptoms and quality of life by increasing $\mathrm{Hb}$. In view of the potential therapeutic effect of EPO in CKD and other anemic diseases, we consider that patients should be administered, combined with anemia-induced diseases, preoperative EPO. In consideration of potential risks like tumorigenesis, patients with tumor should be more cautious in choosing methods of blood management. Future studies will require more detailed stratification of the populations and better formulation of dosing regimens based on EPO's own physiological characteristics.

\section{Limitations}

This meta-analysis has some limitations. The first limitation was the heterogeneity of the included trials was relatively

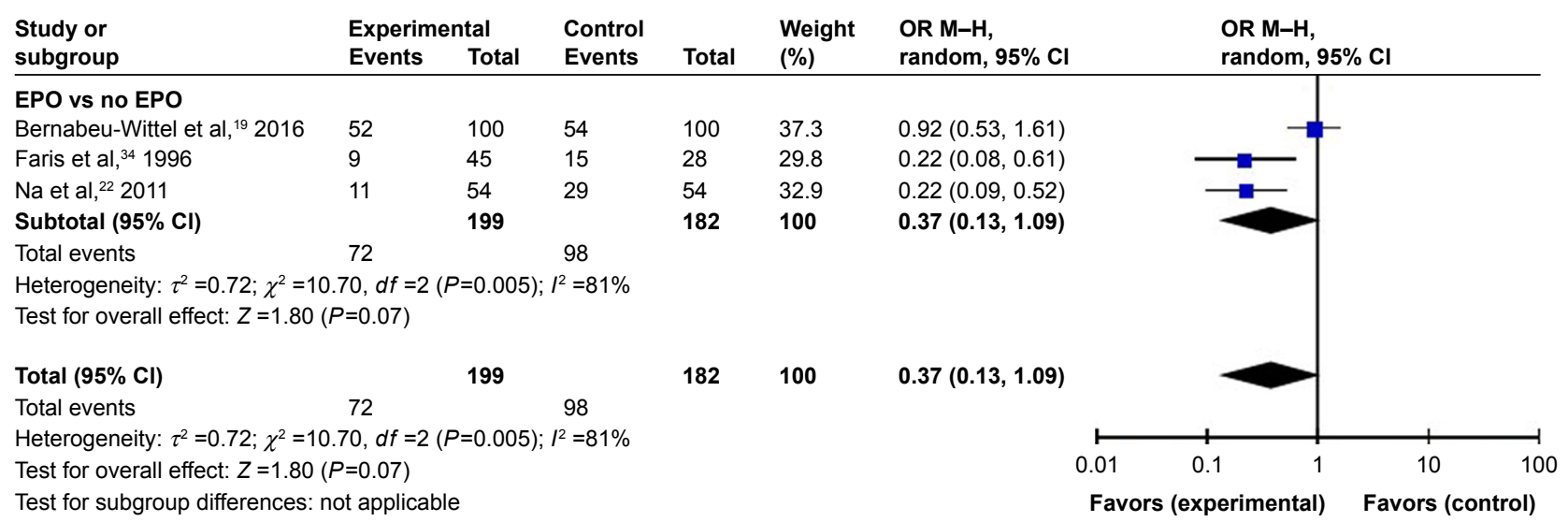

Figure 9 TKA patients needing ABT.

Abbreviations: ABT, allogeneic blood transfusion; EPO, erythropoietin; M-H, Mantel-Haenszel; OR, odds ratio; PABD, preoperative autologous blood donation; TKA, total knee arthroplasty. 


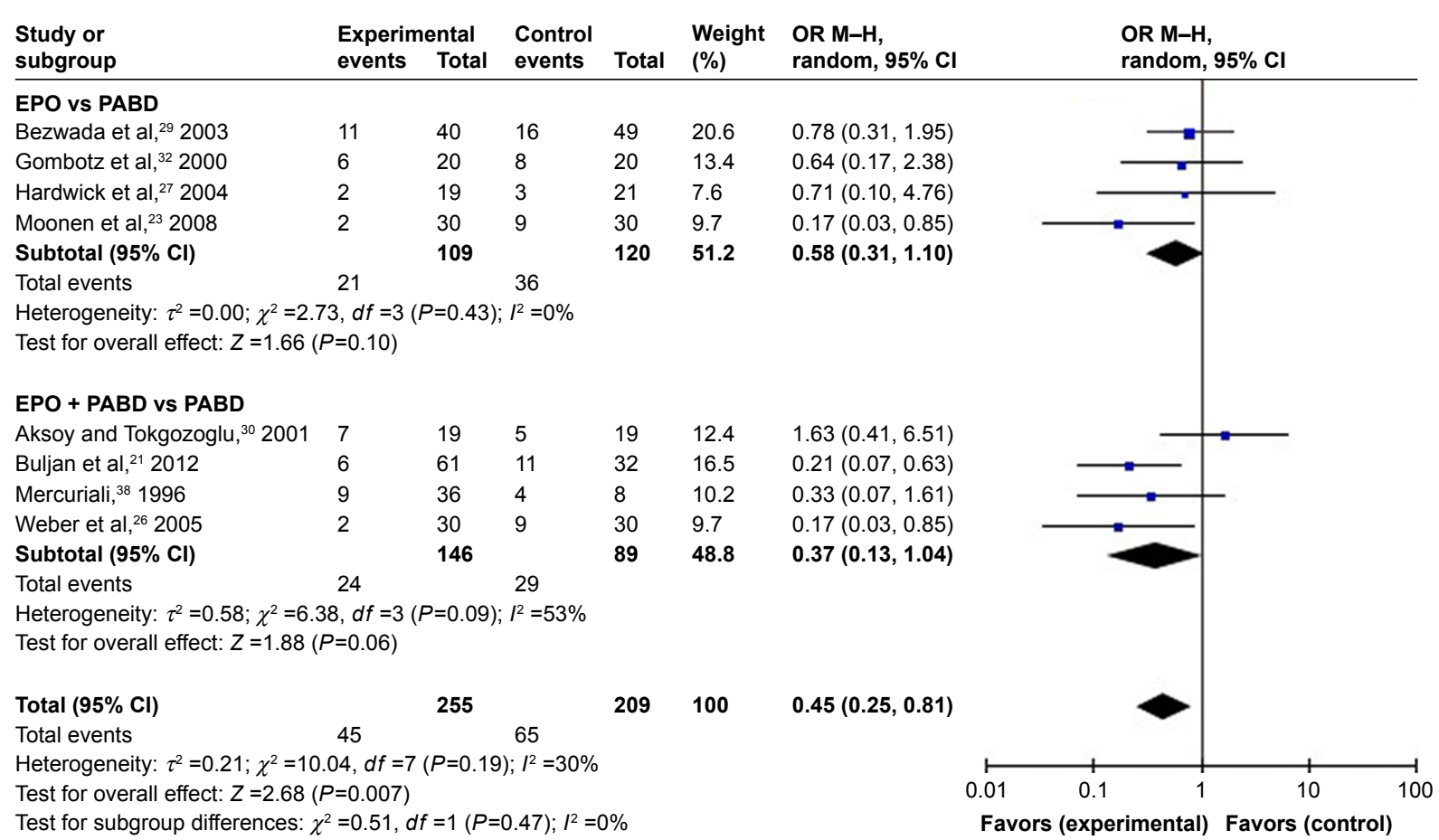

Figure 10 THA patients needing ABT.

Abbreviations: ABT, allogeneic blood transfusion; EPO, erythropoietin; $\mathrm{M}-\mathrm{H}$, Mantel-Haenszel; OR, odds ratio; PABD, preoperative autologous blood donation; THA, total hip arthroplasty.

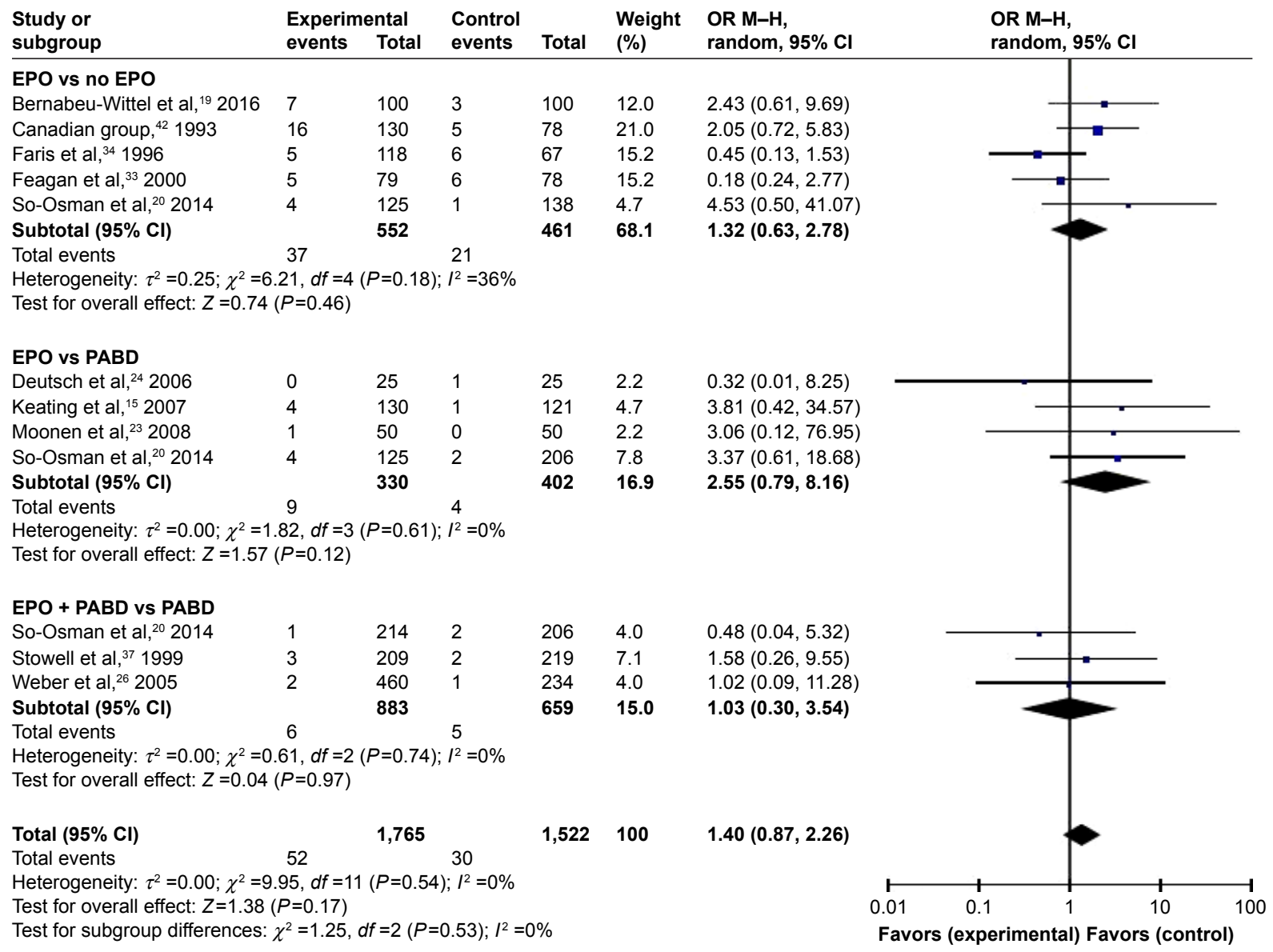

Figure I I The risk of VTE.

Abbreviations: EPO, erythropoietin; M-H, Mantel-Haenszel; OR, odds ratio; PABD, preoperative autologous blood donation; VTE, venous thromboembolism. 
high. After analysis of the subgroups, the heterogeneity decreased to some degree. As $\mathrm{Hb}$ values show a great diversity in normal people, we deduce that this may contribute to the high heterogeneity. Meanwhile, the different type of surgeries and treatment plans may be another reason. After grouping by operation type, TKA and THA, we analyzed the number of patients that received ABT. But there were no enough data to make further analysis. The second limitation was the lack of detailed data for each RCT, which resulted in insufficient data to investigate the impact of EPO on the postoperative recovery or surgical outcomes. The third limitation was the lack of cost analysis because only 2 RCTs concluded the precise information about the cost of the different strategies. However, optimal dosing and duration of EPO therapy remains uncertain as there are potentially important modifiers to its efficacy such as postoperative inflammation and the availability of iron stores. Iron supplementation is indeed a commonly used method during the perioperative period. When we extracted the literature data, we originally planned to conduct a subgroup analysis on iron supplementation. However, the iron supplementation methods and protocols used in different studies differ greatly. Therefore, it is difficult to perform subgroup analysis. Fortunately, each experiment had chosen the same iron supplementation protocol for experimental and control groups. As a result, iron supplementation will not affect the analysis of EPO. Since this study was designed to mainly focus on the role of EPO, iron supplementation was not analyzed. Further studies can be designed to investigate whether iron supplementation would affect the therapeutic effect of EPO. Although iron supplementation is a commonly used method during the perioperative period, many studies did not show whether iron supplementation would have an influence on the effects of EPO. Also, the dosage of iron was not clarified in most of the included studies. Further studies on this issue are needed.

\section{Conclusion}

Preoperative use of EPO can increase pre- and postoperative $\mathrm{Hb}$ levels and decrease the need of ABT in patients undergoing THA or TKA. The effect of EPO is better than using PABD alone, and the combined use of EPO and PABD exerts the best effect in reducing the risk of exposure of ABT than using PABD alone. Further studies should focus on the appropriate perioperative blood management of TKA and THA.

\section{Acknowledgment}

We thank Mackenzie Lucero for manuscript polishing and grammar checking.

\section{Author contributions}

Yi Li and Pengbin Yin performed the data analysis and wrote the article. Houchen Lv and Yutong Meng collected the data and contributed toward designing the study. Licheng Zhang and Peifu Tang designed the study and contributed toward critically revising the paper. All authors contributed toward data analysis, drafting and critically revising the paper and agree to be accountable for all aspects of the work.

\section{Disclosure}

The authors report no conflicts of interest in this work.

\section{References}

1. Tran DHD, Wong GT, Chee YE, Irwin MG. Effectiveness and safety of erythropoiesis-stimulating agent use in the perioperative period. Expert Opin Biol Ther. 2014;14(1):51-61.

2. Zhao Y, Jiang C, Peng H, Feng B, Li Y, Weng X. The effectiveness and safety of preoperative use of erythropoietin in patients scheduled for total hip or knee arthroplasty. Medicine. 2016;95(27):e4122.

3. Voorn VMA, van der Hout A, So-Osman C, et al. Erythropoietin to reduce allogeneic red blood cell transfusion in patients undergoing total hip or knee arthroplasty. Vox Sang. 2016;111(3):219-225.

4. Alsaleh K, Alotaibi GS, Almodaimegh HS, Aleem AA, Kouroukis CT. The use of preoperative erythropoiesis-stimulating agents (ESAs) in patients who underwent knee or hip arthroplasty. J Arthroplasty. 2013; 28(9): 1463-1472.

5. Stivelman JC. Target-based anemia management with erythropoiesis stimulating agents (risks and benefits relearned) and iron (still more to learn). Semin Dial. 2017;30(2):142-148.

6. Elliott S, Tomita D, Endre Z. Erythropoiesis stimulating agents and reno-protection: a meta-analysis. BMC Nephrol. 2017;18(1):14.

7. Foley RN. Emerging erythropoiesis-stimulating agents. Nat Rev Nephrol. 2010;6(4):218-223.

8. Mares JM, Foley LS, Bell MT, et al. Erythropoietin activates the phosporylated cAMP [adenosine $3^{\prime} 5^{\prime}$ cyclic monophosphate] response element-binding protein pathway and attenuates delayed paraplegia after ischemia-reperfusion injury. J Thorac Cardiovasc Surg. 2015; 149(3):920-924.

9. Yamanaka K, Eldeiry M, Aftab M, et al. Optimized induction of beta common receptor enhances the neuroprotective function of erythropoietin in spinal cord ischemic injury. $J$ Thorac Cardiovasc Surg. Epub February 9, 2018.

10. Smith PD, Puskas F, Fullerton DA, et al. Attenuation of spinal cord ischemia and reperfusion injury by erythropoietin. J Thorac Cardiovasc Surg. 2011;141(1):256-260.

11. Robertson CS, Hannay HJ, Yamal JM, et al. Effect of erythropoietin and transfusion threshold on neurological recovery after traumatic brain injury. JAMA. 2014;312(1):36.

12. Gonzalez-Porras JR, Colado E, Conde MP, Lopez T, Nieto MJ, Corral M. An individualized pre-operative blood saving protocol can increase pre-operative haemoglobin levels and reduce the need for transfusion in elective total hip or knee arthroplasty. Transfus Med. 2009;19(1): $35-42$.

13. Hill GE, Frawley WH, Griffith KE, Forestner JE, Minei JP. Allogeneic blood transfusion increases the risk of postoperative bacterial infection: a meta-analysis. J Trauma. 2003;54(5):908-914.

14. Keating EM, Ritter MA. Transfusion options in total joint arthroplasty. J Arthroplasty. 2002;17(4 Suppl 1):125-128.

15. Keating EM, Callaghan JJ, Ranawat AS, Bhirangi K, Ranawat CS. A randomized, parallel-group, open-label trial of recombinant human erythropoietin vs preoperative autologous donation in primary total joint arthroplasty. J Arthroplasty. 2007;22(3):325-333. 
16. Jans $\varnothing$, Jørgensen C, Kehlet H, Johansson PI; Lundbeck Foundation Centre for Fast-track Hip and Knee Replacement Collaborative Group. Role of preoperative anemia for risk of transfusion and postoperative morbidity in fast-track hip and knee arthroplasty. Transfusion. 2014;54(3):717-726.

17. Jorgensen CC, Kehlet H; Lundbeck Foundation Centre for Fast-track Hip and Knee replacement collaborative group. Early thromboembolic events $\leq 1$ week after fast-track total hip and knee arthroplasty. Thromb Res. 2016;138:37-42.

18. Anderson FA Jr, Spencer FA. Risk factors for venous thromboembolism. Circulation. 2003;107(90231):I9-I16

19. Bernabeu-Wittel M, Romero M, Ollero-Baturone M, et al. Ferric carboxymaltose with or without erythropoietin in anemic patients with hip fracture: a randomized clinical trial. Transfusion. 2016;56(9): 2199-2211.

20. So-Osman C, Nelissen RG, Koopman-van Gemert AW, et al. Patient blood management in elective total hip- and knee-replacement surgery (Part 1): a randomized controlled trial on erythropoietin and blood salvage as transfusion alternatives using a restrictive transfusion policy in erythropoietin-eligible patients. Anesthesiology. 2014; 120(4):839-851.

21. Buljan M, Nemet D, Golubic-Cepulic B, Bicanic G, Tripkovic B, Delimar D. Two different dosing regimens of human recombinant erythropoietin beta during preoperative autologous blood donation in patients having hip arthroplasty. Int Orthop. 2012;36(4):703-709.

22. Na HS, Shin SY, Hwang JY, Jeon YT, Kim CS, Do SH. Effects of intravenous iron combined with low-dose recombinant human erythropoietin on transfusion requirements in iron-deficient patients undergoing bilateral total knee replacement arthroplasty (CME). Transfusion. 2011;51(1):118-124.

23. Moonen AF, Thomassen BJ, Knoors NT, van Os JJ, Verburg AD, Pilot P. Pre-operative injections of epoetin-alpha versus post-operative retransfusion of autologous shed blood in total hip and knee replacement: a prospective randomised clinical trial. J Bone Joint Surg Br. 2008;90(8):1079-1083.

24. Deutsch A, Spaulding J, Marcus RE. Preoperative epoetin alfa vs autologous blood donation in primary total knee arthroplasty. J Arthroplasty. 2006;21(5):628-635.

25. Rosencher N, Poisson D, Albi A, Aperce M, Barré J, Samama CM. Two injections of erythropoietin correct moderate anemia in most patients awaiting orthopedic surgery. Can J Anaesth. 2005;52(2):160-165.

26. Weber EW, Slappendel R, Hémon Y, et al. Effects of epoetin alfa on blood transfusions and postoperative recovery in orthopaedic surgery: the European Epoetin Alfa Surgery Trial (EEST). Eur J Anaesthesiol. 2005;22(4):249-257.

27. Hardwick ME, Morris BM, Colwell CW Jr. Two-dose epoetin alfa reduces blood transfusions compared with autologous donation. Clin Orthop Relat Res. 2004;423:240-244.

28. Avall A, Hyllner M, Bengtson JP, Carlsson L, Bengtsson A. Recombinant human erythropoietin in preoperative autologous blood donation did not influence the haemoglobin recovery after surgery. Acta Anaesthesiol Scand. 2003;47(6):687-692.
29. Bezwada HP, Nazarian DG, Henry DH, Booth RE Jr. Preoperative use of recombinant human erythropoietin before total joint arthroplasty. J Bone Joint Surg Am. 2003;85-A(9):1795-1800.

30. Aksoy MC, Tokgozoglu AM. Erythropoietin for autologous blood donation in total hip arthroplasty patients. Arch Orthop Trauma Surg. 2001;121(3):162-165.

31. Olijhoek G, Megens JG, Musto P, et al. Role of oral versus IV iron supplementation in the erythropoietic response to $\mathrm{HH}$ EPO: a randomized, placebo-controlled trial. Transfusion. 2001;41(7):957-963.

32. Gombotz H, Gries M, Sipurzynski S, Fruhwald S, Rehak P. Preoperative treatment with recombinant human erythropoietin or predeposit of autologous blood in women undergoing primary hip replacement. Acta Anaesthesiol Scand. 2000;44(6):737-742.

33. Feagan BG, Wong CJ, Kirkley A, et al. Erythropoietin with iron supplementation to prevent allogeneic blood transfusion in total hip joint arthroplasty. A randomized, controlled trial. Ann Intern Med. 2000;133(11):845-854.

34. Faris PM, Ritter MA, Abels RI. The effects of recombinant human erythropoietin on perioperative transfusion requirements in patients having a major orthopaedic operation. J Bone Joint Surg Am. 1996;78(1): $62-72$.

35. Hasegawa Y, Takamatsu J, Iwase T, Iwasada S, Kitamura S, Iwata H. Effects of recombinant human erythropoietin on thrombosis and fibrinolysis in autologous transfusion for hip surgery. Arch Orthop Trauma Surg. 1999;119(7-8):384-387.

36. Wurnig C, Schatz K, Noske H, et al. Subcutaneous low-dose epoetin beta for the avoidance of transfusion in patients scheduled for elective surgery not eligible for autologous blood donation. Eur Surg Res. 2001;33(5-6):303-310.

37. Stowell CP, Chandler H, Jové M, Guilfoyle M, Wacholtz MC. An open-label, randomized study to compare the safety and efficacy of perioperative epoetin alfa with preoperative autologous blood donation in total joint arthroplasty. Orthopedics. 1999;22(Suppl 1):s105-s112.

38. Mercuriali F. Epoetin alfa increases the volume of autologous blood donated by patients scheduled to undergo orthopedic surgery. Semin Hematol. 1996;33(2 Suppl 2):10-14.

39. Price TH, Goodnough LT, Vogler WR, et al. The effect of recombinant human erythropoietin on the efficacy of autologous blood donation in patients with low hematocrits: a multicenter, randomized, double-blind, controlled trial. Transfusion. 1996;36(1):29-36.

40. Goodnough LT, Price TH, Friedman KD, et al. A phase III trial of recombinant human erythropoietin therapy in nonanemic orthopedic patients subjected to aggressive removal of blood for autologous use: dose, response, toxicity, and efficacy. Transfusion. 1994;34(1):66-71.

41. Beris P, Mermillod B, Levy G, et al. Recombinant human erythropoietin as adjuvant treatment for autologous blood donation. A prospective study. Vox Sang. 1993;65(3):212-218.

42. Effectiveness of perioperative recombinant human erythropoietin in elective hip replacement. Canadian Orthopedic Perioperative Erythropoietin Study Group. Lancet. 1993;341(8855):1227-1232.
Therapeutics and Clinical Risk Management

\section{Publish your work in this journal}

Therapeutics and Clinical Risk Management is an international, peerreviewed journal of clinical therapeutics and risk management, focusing on concise rapid reporting of clinical studies in all therapeutic areas, outcomes, safety, and programs for the effective, safe, and sustained use of medicines. This journal is indexed on PubMed Central, CAS,

\section{Dovepress}

EMBase, Scopus and the Elsevier Bibliographic databases. The manuscript management system is completely online and includes a very quick and fair peer-review system, which is all easy to use. Visit http://www.dovepress.com/testimonials.php to read real quotes from published authors. 